\title{
Homoclinic solutions for a second-order $p$-Laplacian functional differential system with local condition
}

Shiping $\mathrm{Lu}^{1 *}$ and Ming $\mathrm{Lu}^{2}$

\section{"Correspondence:}

lushiping88@sohu.com

${ }^{1}$ College of Math and Statistics,

Nanjing University of Information

Science \& Technology, Nanjing,

210044, China

Full list of author information is

available at the end of the article

\begin{abstract}
By means of critical point theory and some analysis methods, the existence of homoclinic solutions for the $p$-Laplacian system with delay, $\frac{d}{d t}\left[\left|u^{\prime}(t)\right|^{p-2} u^{\prime}(t)\right]=$ $\nabla_{x} G(t, u(t), u(t+\tau))+\nabla_{y} G(t-\tau, u(t-\tau), u(t))+e(t)$, is investigated. Some new results are obtained. The interesting thing is that the function $G(t, x, y)$ is only required to satisfy a local condition. Furthermore, the results are all explicitly related to the value of delay $\tau$.

MSC: $34 \mathrm{C} 37 ; 58 \mathrm{E} 05 ; 70 \mathrm{H} 05$

Keywords: critical point theory; homoclinic solution; periodic solution; functional differential system
\end{abstract}

\section{Introduction}

In the past years, the existence of homoclinic solutions to some second-order ordinary differential systems has been extensively investigated because of its background in applied science (see [1-7] and the references cited therein). For example, in [7], XH Tan and Li Xiao studied the existence of homoclinic solutions to the $p$-Laplacian system

$$
\frac{d}{d t}\left[\left|u^{\prime}(t)\right|^{p-2} u^{\prime}(t)\right]=\nabla F(t, u(t))+f(t)
$$

where $p>1$ is a constant. The following theorem was obtained.

Theorem 1.1 (See [7]) Assume that $F$ and $f$ satisfy the following conditions:

( $\left.\mathrm{B}_{1}\right) F \in C^{1}\left(R \times R^{n}, R\right)$ is $T$-periodic with respect to $t, T>0$ is a constant;

$\left(\mathrm{B}_{2}\right)$ There are constants $b>0$ and $\mu>1$ such that for all $(t, x) \in[0, T] \times R^{n}$

$$
F(t, x) \geq F(t, 0)+b|x|^{\mu}
$$

$\left(\mathrm{B}_{3}\right) f \not \equiv 0$ is a continuous and bounded function such that $\int_{R} \mid f(t)^{\mu /(\mu-1)} d t<+\infty$.

Then (1.1) possesses a homoclinic solution.

From the proof of Theorem 1.1 in [7], we see that assumption $\left(B_{2}\right)$ is crucial for obtaining the existence of a homoclinic solution for (1.1).

\section{Springer}

๑2014 Lu and Lu; licensee Springer. This is an Open Access article distributed under the terms of the Creative Commons Attribution License (http://creativecommons.org/licenses/by/2.0), which permits unrestricted use, distribution, and reproduction in any medium, provided the original work is properly cited. 
However, few papers investigated the existence of homoclinic solutions to functional differential equations [8-10]. In [9], the authors studied the existence of homoclinic solutions to the following functional differential equation:

$$
q^{\prime \prime}(t-\tau)+f(t, q(t), q(t-\tau), q(t-2 \tau))=0,
$$

where $\tau>0$ is a constant, $t \in R, q \in R^{n}, f\left(t, u_{1}, u_{2}, u_{3}\right) \in C\left(R \times R^{n} \times R^{n} \times R^{n}, R^{n}\right)$ and being $\tau$-periodic in $t$. Under

$\left[\mathrm{H}_{1}\right]$ there is a $\tau$-periodic continuously differentiable function $F(t, x, y) \in C^{1}\left(R \times R^{n} \times R^{n} \times\right.$ $\left.R^{n}, R\right)$ such that

$$
\nabla_{x} F(t, q(t-\tau), q(t-2 \tau))+\nabla_{y} F(t, q(t), q(t-\tau))=f(t, q(t), q(t-\tau), q(t-2 \tau))
$$

$\left[\mathrm{H}_{2}\right]$ there is a $e \in R^{n}$ with $e \neq 0$ such that

$$
F_{0}=\limsup _{|v| \rightarrow \infty} F\left(t, v_{1}, v_{2}\right)<0 \quad \text { for all } t \in R
$$

and some other conditions, they obtained the result that (1.2) possesses a nontrivial homoclinic orbit.

In this paper, we investigate further the existence of homoclinic solutions for a secondorder $p$-Laplacian functional differential system as follows:

$$
\frac{d}{d t}\left[\left|u^{\prime}(t)\right|^{p-2} u^{\prime}(t)\right]=\nabla_{x} G(t, u(t), u(t+\tau))+\nabla_{y} G(t-\tau, u(t-\tau), u(t))+e(t),
$$

where $u(t)=\left(u_{1}(t), u_{2}(t), \ldots, u_{n}(t)\right)^{\top} \in R^{n}, t \in R, G \in C^{1}\left(R \times R^{n} \times R^{n}, R\right)$ and is $T$-periodic in $t, \nabla_{x} G(t, x, y)=\frac{\partial G(t, x, y)}{\partial x}, \nabla_{y} G(t, x, y)=\frac{\partial G(t, x, y)}{\partial y}, e \in C\left(R, R^{n}\right), \tau, T>0$ and $p>1$ are constants. The interesting thing of this paper is that the period $T$ of function $f\left(t, u_{1}, u_{2}, u_{3}\right)$ with respect to the variable $t$ may not be equal to $\tau$, and the main results are all expressively related to the value of delay $\tau$. Furthermore, even if for the case of $\tau=0$, we do not require condition $\left(\mathrm{B}_{2}\right)$ for guaranteeing the coercive potential condition.

As is well known, a solution $u(t)$ of (1.3) is named homoclinic (to 0 ) if $u(t) \rightarrow 0$ and $u^{\prime}(t) \rightarrow 0$ as $|t| \rightarrow+\infty$. In addition, if $u \neq \equiv$, then $u$ is called a nontrivial homoclinic solution.

Motivated by the idea in the work of PH Rabinowitz in [5], Marek Izydorek, Joanna Janczewska in [6] and XH Tan, Li Xiao in [7], the existence of a homoclinic solution for (1.5) is obtained as a limit of a certain sequence $\left\{u_{k_{j}}(t)\right\}_{j \in \mathbf{N}}$ of $2 k T$-periodic solutions for the following equation:

$$
\frac{d}{d t}\left[\left|u^{\prime}(t)\right|^{p-2} u^{\prime}(t)\right]=\nabla_{x} G(t, u(t), u(t+\tau))+\nabla_{y} G(t-\tau, u(t-\tau), u(t))+e_{k}(t),
$$

where $k \in \mathbf{N}, e_{k}: R \rightarrow R^{n}$ is a $2 k T$-periodic extension of restriction of $e$ to the interval $[-k T, k T]$. We obtain the following theorem.

Theorem 1.2 Assume that $G$ and e satisfy the following conditions:

$\left(C_{1}\right) \nabla_{x} G(t, 0,0)+\nabla_{y} G(t-\tau, 0,0)=0$ for all $t \in R$; 
$\left(C_{2}\right)$ there are constants $b_{1}>0, b_{i} \geq 0(i=2,3,4), \mu>0$, and $1<\lambda<p$ such that

$$
\begin{aligned}
G(t, x, y) \geq & G(t, 0,0)+b_{1}|x|^{\mu}-b_{2}|y|^{\mu}-b_{3}|x-y|^{p} \\
& -b_{4}|x-y|^{\lambda}\left(|x|^{\frac{(p-\lambda) \mu}{p}}+|y|^{\frac{(p-\lambda) \mu}{p}}\right)
\end{aligned}
$$

for all $(t, x, y) \in R \times R^{n} \times R^{n}$ with $|x| \leq \rho,|y| \leq \rho$;

or

$$
\begin{gathered}
G(t, x, y) \geq G(t, 0,0)+b_{1}|y|^{\mu}-b_{2}|x|^{\mu}-b_{3}|x-y|^{p} \\
\quad-b_{4}|x-y|^{\lambda}\left(|x|^{\frac{(p-\lambda) \mu}{p}}+|y|^{\frac{(p-\lambda) \mu}{p}}\right) \\
\text { for all }(t, x, y) \in R \times R^{n} \times R^{n} \text { with }|x| \leq \rho,|y| \leq \rho,
\end{gathered}
$$

where $\rho$ is a constant with $\rho>\frac{\mu-1}{\mu} T^{\frac{1-\mu}{\mu}}+\frac{T(p-1)}{p}$;

$\left(C_{3}\right) e \neq \equiv$ is a continuous and bounded function such that $M:=\left(\int_{R}|e(t)|^{\mu /(\mu-1)} d t\right)^{\frac{\mu-1}{\mu}}<$ $+\infty$.

Then (1.3) possesses a nontrivial homoclinic solution, if

$$
b_{1}-b_{2}-\frac{2 b_{4}(p-\lambda)}{p} \tau^{\lambda}>0
$$

and

$$
\begin{gathered}
\min \left\{\frac{1}{p}-b_{3} \tau^{p}-\frac{2 b_{4} \lambda}{p} \tau^{\lambda}, \frac{1}{2}\left(b_{1}-b_{2}-\frac{2 b_{4}(p-\lambda)}{p} \tau^{\lambda}\right)\right\} \frac{\rho-\frac{\mu-1}{\mu} T^{\frac{1-\mu}{\mu}}-\frac{T(p-1)}{p}}{\max \left\{\frac{1}{\mu}, \frac{1}{p}\right\}} \\
-\frac{1}{2}\left(b_{1}-b_{2}-\frac{2 b_{4}(p-\lambda)}{p} \tau^{\lambda}\right)(\mu-1)\left(\frac{2 M}{\left(b_{1}-b_{2}-\frac{2 b_{4}(p-\lambda)}{p} \tau^{\lambda}\right) \mu}\right)^{\frac{\mu}{\mu-1}}>0 .
\end{gathered}
$$

In particular, suppose

$$
\begin{aligned}
G(t, x, y) \geq & G(t, 0,0)+b_{1}|x|^{\mu}-b_{2}|y|^{\mu} \\
& -\sum_{j=1}^{l} c_{j}|x|^{m_{j}}-b_{3}|x-y|^{p}-b_{4}|x-y|^{\lambda}\left(|x|^{\frac{(p-\lambda) \mu}{p}}+|y|^{\frac{(p-\lambda) \mu}{p}}\right)
\end{aligned}
$$

for all $(t, x, y) \in R \times R^{n} \times R^{n}$;

or

$$
\begin{aligned}
G(t, x, y) \geq & G(t, 0,0)+b_{1}|y|^{\mu}-b_{2}|x|^{\mu} \\
& -\sum_{j=1}^{l} c_{j}|y|^{m_{j}}-b_{3}|x-y|^{p}-b_{4}|x-y|^{\lambda}\left(|x|^{\frac{(p-\lambda) \mu}{p}}+|y|^{\frac{(p-\lambda) \mu}{p}}\right)
\end{aligned}
$$

for all $(t, x, y) \in R \times R^{n} \times R^{n}$, 
where $b_{i}>0, i=1,2, \ldots, 4, c_{j}>0$, and $m_{j}>\mu>1, j=1,2, \ldots, l$, are all constants. If there is a constant $\rho>\frac{\mu-1}{\mu} T^{\frac{1-\mu}{\mu}}+\frac{T(p-1)}{p}$ such that

$$
\delta:=b_{1}-\sum_{j=1}^{l} c_{j} \rho^{m_{j}-\mu}>0,
$$

then from (1.6), one can easily find

$$
\begin{aligned}
& G(t, x, y) \geq G(t, 0,0)+\delta|x|^{\mu}-b_{2}|y|^{\mu}-b_{3}|x-y|^{p}-b_{4}|x-y|^{\lambda}\left(|x|^{\frac{(p-\lambda) \mu}{p}}+|y|^{\frac{(p-\lambda) \mu}{p}}\right) \\
& \quad \text { for all }(t, x, y) \in R \times R^{n} \times R^{n} \text { with }|x| \leq \rho,|y| \leq \rho ;
\end{aligned}
$$

and from (1.7), we have

$$
\begin{aligned}
& G(t, x, y) \geq G(t, 0,0)+\delta|y|^{\mu}-b_{2}|x|^{\mu}-b_{3}|x-y|^{p}-b_{4}|x-y|^{\lambda}\left(|x|^{\frac{(p-\lambda) \mu}{p}}+|y|^{\frac{(p-\lambda) \mu}{p}}\right) \\
& \quad \text { for all }(t, x, y) \in R \times R^{n} \times R^{n} \text { with }|x| \leq \rho,|y| \leq \rho .
\end{aligned}
$$

So by using Theorem 1.2, we have the following result.

Corollary 1.1 Assume that assumption $\left(\mathrm{C}_{1}\right)$, assumption $\left(\mathrm{C}_{3}\right)$, condition (1.6) (or condition (1.7)) and condition (1.8) hold. Then (1.3) possesses a nontrivial homoclinic solution, if

$$
\delta-b_{2}-\frac{2 b_{4}(p-\lambda)}{p} \tau^{\lambda}>0
$$

and

$$
\begin{aligned}
\min & \left\{\frac{1}{p}-b_{3} \tau^{p}-\frac{2 b_{4} \lambda}{p} \tau^{\lambda}, \frac{1}{2}\left(\delta-b_{2}-\frac{2 b_{4}(p-\lambda)}{p} \tau^{\lambda}\right)\right\} \frac{\rho-\frac{\mu-1}{\mu} T^{\frac{1-\mu}{\mu}}-\frac{T(p-1)}{p}}{\max \left\{\frac{1}{\mu}, \frac{1}{p}\right\}} \\
& -\frac{1}{2}\left(\delta-b_{2}-\frac{2 b_{4}(p-\lambda)}{p} \tau^{\lambda}\right)(\mu-1)\left(\frac{2 M}{\left(\delta-b_{2}-\frac{2 b_{4}(p-\lambda)}{p} \tau^{\lambda}\right) \mu}\right)^{\frac{\mu}{\mu-1}}>0 .
\end{aligned}
$$

Remark 1.1 If $b_{1}>b_{2}$, and assumptions $\left(C_{1}\right)-\left(C_{3}\right)$ are satisfied, then for sufficiently small $\tau>0$ and sufficiently large $\rho>0$, we see that condition (1.5) is satisfied. So by using Theorem 1.2, we see that (1.3) possesses a nontrivial homoclinic solution. Furthermore, if $\tau=0$, then (1.3) is converted to

$$
\frac{d}{d t}\left[\left|u^{\prime}(t)\right|^{p-2} u^{\prime}(t)\right]=\nabla H(t, u(t))+e(t),
$$

where $H(t, u(t))=G(t, u(t), u(t))$, and from (1.6) or (1.7), we see that the function $H(t, x)$ is allowed to be

$$
H(t, x)-H(t, 0)=G(t, x, x)-G(t, 0,0) \rightarrow-\infty \quad \text { as }|x| \rightarrow+\infty,
$$

which implies condition $\left(B_{2}\right)$ for guaranteeing the coercive potential does not hold for (1.3). Moreover, we do not require that $G(t, x, y)$ is $\tau$-periodic function with respect to $t$, which is required by [9], and local condition $\left(C_{2}\right)$ is essentially different from assumption $\left[\mathrm{H}_{2}\right]$ in [9]. 
If $\mu$ in assumption $\left(C_{2}\right)$ is the case $\mu=p$, then we have further the following result.

Theorem 1.3 Assume that assumption $\left(\mathrm{C}_{1}\right)$ in Theorem 1.2 is satisfied together with the following conditions:

$\left(\mathrm{D}_{2}\right)$ there are constants $\rho>0, b_{1}>0, b_{i}>0(i=2,3,4), \mu>0$ and $1<\lambda<p$ such that for all $(t, x, y) \in R \times R^{n} \times R^{n}$ with $|x| \leq \rho,|y| \leq \rho$,

$$
\begin{aligned}
G(t, x, y) \geq & G(t, 0,0)+b_{1}|x|^{p}-b_{2}|y|^{p}-b_{3}|x-y|^{p} \\
& -b_{4}|x-y|^{\lambda}\left(|x|^{(p-\lambda)}+|y|^{(p-\lambda)}\right)
\end{aligned}
$$

or

$$
\begin{gathered}
G(t, x, y) \geq G(t, 0,0)+b_{1}|y|^{p}-b_{2}|x|^{p}-b_{3}|x-y|^{p} \\
-b_{4}|x-y|^{\lambda}\left(|x|^{(p-\lambda)}+|y|^{(p-\lambda)}\right) ;
\end{gathered}
$$

$\left(\mathrm{D}_{3}\right) e \not \equiv 0$ is a continuous and bounded function such that $M_{1}:=\left(\int_{R}|e(t)|^{p /(p-1)} d t\right)^{\frac{p-1}{p}}<$ $+\infty$.

Then (1.3) possesses a nontrivial homoclinic solution, if

$$
b_{1}-b_{2}-\frac{2 b_{4}(p-\lambda)}{p} \tau^{\lambda}>0
$$

and

$$
\begin{gathered}
\min \left\{\frac{1}{p}-b_{3} \tau^{p}-\frac{2 b_{4} \lambda}{p} \tau^{\lambda}, \frac{1}{2}\left(b_{1}-b_{2}-\frac{2 b_{4}(p-\lambda)}{p} \tau^{\lambda}\right)\right\} \rho^{p}\left(T^{1-q}+T\right)^{1-p} \\
-\frac{1}{2}\left(b_{1}-b_{2}-\frac{2 b_{4}(p-\lambda)}{p} \tau^{\lambda}\right)(p-1)\left(\frac{2 M_{1}}{\left(b_{1}-b_{2}-\frac{2 b_{4}(p-\lambda)}{p} \tau^{\lambda}\right) p}\right)^{\frac{p}{p-1}}>0 .
\end{gathered}
$$

Suppose there are constants $b_{1}>0, b_{i}>0(i=2,3,4), c_{j}>0, m_{j}>p(j=1,2, \ldots, l), \mu>0$, and $1<\lambda<p$ such that for all $(t, x, y) \in R \times R^{n} \times R^{n}$,

$$
\begin{aligned}
G(t, x, y) \geq & G(t, 0,0)+b_{1}|x|^{p}-b_{2}|y|^{p} \\
& -\sum_{j=1}^{l} c_{j}|x|^{m_{j}}-b_{3}|x-y|^{p}-b_{4}|x-y|^{\lambda}\left(|x|^{(p-\lambda)}+|y|^{(p-\lambda)}\right)
\end{aligned}
$$

or

$$
\begin{aligned}
G(t, x, y) \geq & G(t, 0,0)+b_{1}|y|^{p}-b_{2}|x|^{p} \\
& -\sum_{j=1}^{l} c_{j}|y|^{m_{j}}-b_{3}|x-y|^{p}-b_{4}|x-y|^{\lambda}\left(|x|^{(p-\lambda)}+|y|^{(p-\lambda)}\right) .
\end{aligned}
$$


If there is a constant $\rho>0$ such that

$$
\delta_{1}:=b_{1}-\sum_{j=1}^{l} c_{j} \rho^{m_{j}-p}>0,
$$

then for all $(t, x, y) \in R \times R^{n} \times R^{n}$ with $|x| \leq \rho,|y| \leq \rho$,

$$
G(t, x, y) \geq G(t, 0,0)+\delta_{1}|x|^{p}-b_{2}|y|^{p}-b_{3}|x-y|^{p}-b_{4}|x-y|^{\lambda}\left(|x|^{(p-\lambda)}+|y|^{(p-\lambda)}\right)
$$

or

$$
G(t, x, y) \geq G(t, 0,0)+\delta_{1}|y|^{p}-b_{2}|x|^{p}-b_{3}|x-y|^{p}-b_{4}|x-y|^{\lambda}\left(|x|^{(p-\lambda)}+|y|^{(p-\lambda)}\right) .
$$

So by using Theorem 1.3, we have the following result.

Corollary 1.2 Assume that assumption $\left(\mathrm{C}_{1}\right)$ in Theorem 1.2, assumption $\left(\mathrm{D}_{3}\right)$ in Theorem 1.3, condition (1.10) (or condition (1.11)) and condition (1.12) hold. Then (1.3) possesses a nontrivial homoclinic solution, if

$$
\delta_{1}-b_{2}-\frac{2 b_{4}(p-\lambda)}{p} \tau^{\lambda}>0
$$

and

$$
\begin{aligned}
\min & \left\{\frac{1}{p}-b_{3} \tau^{p}-\frac{2 b_{4} \lambda}{p} \tau^{\lambda}, \frac{1}{2}\left(\delta_{1}-b_{2}-\frac{2 b_{4}(p-\lambda)}{p} \tau^{\lambda}\right)\right\} \rho^{p}\left(T^{1-q}+T\right)^{1-p} \\
& -\frac{1}{2}\left(\delta_{1}-b_{2}-\frac{2 b_{4}(p-\lambda)}{p} \tau^{\lambda}\right)(p-1)\left(\frac{2 M_{1}}{\left(\delta_{1}-b_{2}-\frac{2 b_{4}(p-\lambda)}{p} \tau^{\lambda}\right) p}\right)^{\frac{p}{p-1}}>0,
\end{aligned}
$$

where $\delta_{1}$ is determined in (1.12).

\section{Preliminaries}

Throughout this paper, $(\cdot, \cdot): R^{n} \times R^{n} \rightarrow R$ denotes the standard inner product in $R^{n}$ and $|\cdot|$ is the induced norm. For each $k \in \mathbf{N}, E_{k}=W_{2 k T}^{1, p}\left(R, R^{n}\right)$ denotes the Banach space of $2 k T$ periodic functions on $R$ with values in $R^{n}$ under the norm

$$
\|u\|_{E_{k}}:=\left[\int_{-k T}^{k T}\left(\left|u^{\prime}(t)\right|^{p}+|u(t)|^{p}\right) d t\right]^{1 / p}
$$

$L_{2 k T}^{p}\left(R, R^{n}\right)$ denotes the Banach space of $2 k T$-periodic functions on $R$ with values in $R^{n}$ under the norm

$$
\|u\|_{p}=\left[\int_{-k T}^{k T}|u(t)|^{p} d t\right]^{1 / p}
$$

and $L_{2 k T}^{\infty}\left(R, R^{n}\right)$ denotes the Banach space of $2 k T$-periodic essentially bounded measurable functions from $R$ to $R^{n}$ with the norm

$$
\|u\|_{\infty}=\operatorname{ess} \sup \{|u(t)|: t \in[-k T, k T]\} .
$$


Lemma 2.1 $[7,10]$ Let $x \in C^{1}\left(R, R^{n}\right)$ with $\left|\frac{d}{d t}\left[\left|x^{\prime}(t)\right|^{p-2} x^{\prime}(t)\right]\right| \leq R_{1}$ and $\int_{-\infty}^{+\infty}\left|x^{\prime}(s)\right|^{\sigma} d s \leq R_{0}$, where $R_{0}, R_{1}$, and $\sigma$ are positive constants. Then $x^{\prime}(t) \rightarrow 0$ as $t \rightarrow \pm \infty$.

Lemma 2.2 [11] Let $n_{1}>1, \omega>0$ and $\beta \in[0,+\infty)$ be constants, $s \in C(R, R)$ with $s(t) \in[0, \beta)$ or $s(t) \in(-\beta, 0]$. Then for each $x \in C^{1}\left(R, R^{n}\right)$ with $x(t+\omega) \equiv x(t)$, we have

$$
\int_{0}^{\omega}|x(t)-x(t-s(t))|^{n_{1}} d t \leq \beta^{n_{1}} \int_{0}^{\omega}\left|x^{\prime}(t)\right|^{n_{1}} d t
$$

Lemma 2.3 [7] If $q: R \rightarrow R^{n}$ is continuous differential on $R, a>0, \mu>1$ and $p>1$ are constants, then for every $t \in R$ the following inequality holds:

$$
|q(t)| \leq(2 a)^{-\frac{1}{\mu}}\left(\int_{t-a}^{t+a}|q(s)|^{\mu} d s\right)^{1 / \mu}+a(2 a)^{-1 / p}\left(\int_{t-a}^{t+a}\left|q^{\prime}(s)\right|^{p} d s\right)^{1 / p} .
$$

Lemma 2.4 [10] Let $X$ be a real reflexive Banach space and $\Omega \subset X$ be a bounded convex closed subset of $X$. Suppose that $\varphi: X \rightarrow R$ is a lower weakly semi-continuous functional. If there exists a point $x_{0} \in \Omega \backslash \partial \Omega$ such that

$$
\varphi(x)>\varphi\left(x_{0}\right), \quad \forall x \in \partial \Omega,
$$

then there must be $a x^{*} \in \Omega \backslash \partial \Omega$ such that

$$
\varphi\left(x^{*}\right)=\inf _{u \in \Omega} \varphi(u)
$$

In order to investigate the existence of homoclinic solutions to (1.3), we should study the existence of $2 k T$-periodic solutions to (1.4) for each $k \in \mathbf{N}$ in the first case.

Lemma 2.5 Assume that the functions $G$ and e satisfy conditions $\left(\mathrm{C}_{1}\right)-\left(\mathrm{C}_{3}\right)$, and also condition (1.5) holds. Then for each $k \in \mathbf{N}$, (1.4) possesses a $2 k T$-periodic solution $u_{k}(t)$ such that

$$
\left\|u_{k}\right\|_{\mu} \leq A_{0} \text { and }\left\|u_{k}^{\prime}\right\|_{p} \leq A_{1}
$$

where $A_{0}$ and $A_{1}$ are constants independent of $k$.

Proof For each $k \in \mathbf{N}$, let $\varphi_{k}: E_{k} \rightarrow R$ be defined by

$$
\varphi_{k}(u)=\int_{-k T}^{k T}\left[\frac{1}{p}\left|u^{\prime}(t)\right|^{p}+G(t, u(t), u(t+\tau))+\left(e_{k}(t), u(t)\right)\right] d t .
$$

Then $\varphi_{k} \in C^{1}\left(E_{k}, R\right)$ and is weakly lower semi-continuous. Furthermore, one can easily check

$$
\begin{aligned}
\varphi_{k}^{\prime}(u) v= & \int_{-k T}^{k T}\left[\left(\left|u^{\prime}(t)\right|^{p-2} u^{\prime}(t), v^{\prime}(t)\right)+\left(\nabla_{x} G(t, u(t), u(t+\tau)), v(t)\right)\right. \\
& \left.+\left(\nabla_{y} G(t, u(t), u(t+\tau)), v(t+\tau)\right)+\left(e_{k}(t), v(t)\right)\right] d t, \quad \forall v \in E_{k} .
\end{aligned}
$$


Since $\int_{-k T}^{k T}\left(\nabla_{y} G(t, u(t), u(t+\tau)), v(t+\tau)\right) d t=\int_{-k T}^{k T}\left(\nabla_{y} G(t-\tau, u(t-\tau), u(t)), v(t)\right) d t$, it follows from (2.2) that

$$
\begin{aligned}
\varphi_{k}^{\prime}(u) v= & \int_{-k T}^{k T}\left[\left(\left|u^{\prime}(t)\right|^{p-2} u^{\prime}(t), v^{\prime}(t)\right)+\left(\nabla_{x} G(t, u(t), u(t+\tau)), v(t)\right)\right. \\
& \left.+\left(\nabla_{y} G(t-\tau, u(t-\tau), u(t)), v(t)\right)+\left(e_{k}(t), v(t)\right)\right] d t, \quad \forall v \in E_{k} .
\end{aligned}
$$

So if $u_{0} \in E_{k}$ is a critical point of $\varphi_{k}$, then $u_{0}$ must be a $2 k T$-periodic solution to (1.6). Thus, we should prove that $\varphi_{k}$ possesses a critical point. In order to do it, let $C_{0}=\int_{0}^{T} G(t, 0,0) d t$ and $\Omega=\left\{x \in E_{k}: \int_{-k T}^{k T}\left|u^{\prime}(t)\right|^{p} d t+\int_{-k T}^{k T}|u(t)|^{\mu} d t \leq \rho_{1}\right\}$, where

$$
\rho_{1}=\frac{\rho-\frac{\mu-1}{\mu} T^{\frac{1-\mu}{\mu}}-\frac{T(p-1)}{p}}{\max \left\{\frac{1}{\mu}, \frac{1}{p}\right\}},
$$

$\rho>0$ is a constant defined by assumption $\left(\mathrm{C}_{2}\right)$. From [10], we see $\Omega$ is a closed bounded convex subset of $E_{k}$. Now, for using Lemma 2.4, we should prove that for each $k \in \mathbf{N}$,

$$
\varphi_{k}(u)>\varphi_{k}(0), \quad \forall u \in \partial \Omega
$$

If $u \in \partial \Omega$, then $\int_{-k T}^{k T}\left|u^{\prime}(t)\right|^{p} d t+\int_{-k T}^{k T}|u(t)|^{\mu} d t=\rho_{1}$. So by using Lemma 2.3, we have

$$
\begin{aligned}
\|u\|_{L_{2 k T}^{\infty}} & \leq T^{-\frac{1}{\mu}}\left(\int_{-k T}^{k T}|u(s)|^{\mu} d s\right)^{\frac{1}{\mu}}+T^{\frac{p-1}{p}}\left(\int_{-k T}^{k T}\left|u^{\prime}(s)\right|^{p} d s\right)^{\frac{1}{p}} \\
& \leq \frac{1}{\mu} \int_{-k T}^{k T}|u(s)|^{\mu} d s+\frac{1}{p} \int_{-k T}^{k T}\left|u^{\prime}(s)\right|^{p} d s+\frac{\mu-1}{\mu} T^{1-\mu}+\frac{(p-1) T}{p} \\
& \leq \max \left\{\frac{1}{\mu}, \frac{1}{p}\right\} \rho_{1}+\frac{\mu-1}{\mu} T^{1-\mu}+\frac{(p-1) T}{p} .
\end{aligned}
$$

Substituting $\rho_{1}$ in (2.3) into the above formula,

$$
\|u\|_{L_{2 k T}^{\infty}} \leq \rho, \quad \forall u \in \partial \Omega
$$

So, for all $u \in \partial \Omega$, by using conditions $\left(\mathrm{C}_{1}\right)$ and $\left(\mathrm{C}_{2}\right)$,

$$
\begin{aligned}
\varphi_{k}(u)= & \int_{-k T}^{k T}\left[\frac{1}{p}\left|u^{\prime}(t)\right|^{p}+G(t, u(t), u(t+\tau))+\left(e_{k}(t), u(t)\right)\right] d t \\
\geq & \int_{-k T}^{k T}\left[\frac{1}{p}\left|u^{\prime}(t)\right|^{p}+G(t, 0,0)+b_{1}|u(t)|^{\mu}-b_{2}|u(t+\tau)|^{\mu}-b_{3}|u(t)-u(t+\tau)|^{p}\right. \\
& \left.-b_{4}|u(t)-u(t+\tau)|^{\lambda}\left(|u(t)|^{\frac{(p-\lambda) \mu}{p}}+|u(t+\tau)|^{\frac{(p-\lambda) \mu}{p}}\right)+\left(e_{k}(t), u(t)\right)\right] d t \\
= & 2 k C_{0}+\int_{-k T}^{k T}\left[\frac{1}{p}\left|u^{\prime}(t)\right|^{p}+\left(b_{1}-b_{2}\right)|u(t)|^{\mu}+\left(e_{k}(t), u(t)\right)\right] d t \\
& -b_{3} \int_{-k T}^{k T}|u(t)-u(t+\tau)|^{p} d t-b_{4} \int_{-k T}^{k T}|u(t)-u(t+\tau)|^{\lambda}|u(t)|^{\frac{(p-\lambda) \mu}{p}} d t \\
& -b_{4} \int_{-k T}^{k T}|u(t-\tau)-u(t)|^{\lambda}|u(t)|^{\frac{(p-\lambda) \mu}{p}} d t .
\end{aligned}
$$


By using the Hölder inequality and Lemma 2.2,

$$
\begin{aligned}
& \int_{-k T}^{k T}|u(t)-u(t+\tau)|^{\lambda}|u(t)|^{\frac{(p-\lambda) \mu}{p}} d t \\
& \quad \leq\left(\int_{-k T}^{k T}|u(t)-u(t+\tau)|^{p} d t\right)^{\frac{\lambda}{p}}\left(\int_{-k T}^{k T}|u(t)|^{\mu} d t\right)^{\frac{p-\lambda}{p}} \\
& \quad \leq \tau^{\lambda}\left\|u^{\prime}\right\|_{p}^{\lambda}\|u\|^{\frac{(p-\lambda) \mu}{p}}
\end{aligned}
$$

Similarly,

$$
\begin{aligned}
& \int_{-k T}^{k T}|u(t-\tau)-u(t)|^{\lambda}|u(t)|^{\frac{(p-\lambda) \mu}{p}} d t \leq \tau^{\lambda}\left\|u^{\prime}\right\|_{p}^{\lambda}\|u\|^{\frac{(p-\lambda) \mu}{p}} \\
& \int_{-k T}^{k T}|u(t+\tau)-u(t)|^{p} d t \leq \tau^{p}\left\|u^{\prime}\right\|_{p^{\prime}}
\end{aligned}
$$

In view of condition $\left(C_{3}\right)$, we see

$$
\left|\int_{-k T}^{k T}\left(e_{k}(t), u(t)\right) d t\right| \leq M\|u\|_{\mu} .
$$

Substituting (2.5), (2.6), (2.7), and (2.8) into (2.4), and using the Young inequality,

$$
\begin{aligned}
\varphi_{k}(u) & \\
\geq & 2 k C_{0}+\left(\frac{1}{p}-b_{3} \tau^{p}\right)\left\|u^{\prime}\right\|_{p}^{p}+\left(b_{1}-b_{2}\right)\|u\|_{\mu}^{\mu}-2 b_{4} \tau^{\lambda}\left\|u^{\prime}\right\|_{p}^{\lambda}\|u\|_{\mu}^{\frac{(p-\lambda) \mu}{p}}-M\|u\|_{\mu} \\
\geq & 2 k C_{0}+\left(\frac{1}{p}-b_{3} \tau^{p}\right)\left\|u^{\prime}\right\|_{p}^{p}+\left(b_{1}-b_{2}\right)\|u\|_{\mu}^{\mu} \\
& -2 b_{4} \tau^{\lambda}\left(\frac{\lambda}{p}\left\|u^{\prime}\right\|_{p}^{p}+\frac{p-\lambda}{p}\|u\|_{\mu}^{\mu}\right)-M\|u\|_{\mu} \\
= & 2 k C_{0}+\left(\frac{1}{p}-b_{3} \tau^{p}-\frac{2 b_{4} \lambda}{p} \tau^{\lambda}\right)\left\|u^{\prime}\right\|_{p}^{p}+\left(b_{1}-b_{2}-\frac{2 b_{4}(p-\lambda)}{p} \tau^{\lambda}\right)\|u\|_{\mu}^{\mu}-M\|u\|_{\mu} \\
\geq & 2 k C_{0}+\min \left\{\frac{1}{p}-b_{3} \tau^{p}-\frac{2 b_{4} \lambda}{p} \tau^{\lambda}, \frac{1}{2}\left(b_{1}-b_{2}-\frac{2 b_{4}(p-\lambda)}{p} \tau^{\lambda}\right)\right\}\left(\|u\|_{\mu}^{\mu}+\left\|u^{\prime}\right\|_{p}^{p}\right) \\
& +\frac{1}{2}\left(b_{1}-b_{2}-\frac{2 b_{4}(p-\lambda)}{p} \tau^{\lambda}\right)\|u\|_{\mu}^{\mu}-M\|u\|_{\mu} .
\end{aligned}
$$

By using the inequality (see [7])

$$
\frac{B}{2} x^{\mu}-M x \geq-\frac{B}{2}(\mu-1)\left(\frac{2 M}{B \mu}\right)^{\frac{\mu}{\mu-1}}, \quad \forall x \in[0,+\infty)
$$

where $B>0$ is a constant, we obtain from (2.9) the result that

$$
\begin{aligned}
& \varphi_{k}(u) \\
& \qquad \geq \min \left\{\frac{1}{p}-b_{3} \tau^{p}-\frac{2 b_{4} \lambda}{p} \tau^{\lambda}, \frac{1}{2}\left(b_{1}-b_{2}-\frac{2 b_{4}(p-\lambda)}{p} \tau^{\lambda}\right)\right\}\left(\|u\|_{\mu}^{\mu}+\left\|u^{\prime}\right\|_{p}^{p}\right)
\end{aligned}
$$




$$
\begin{aligned}
& -\frac{1}{2}\left(b_{1}-b_{2}-\frac{2 b_{4}(p-\lambda)}{p} \tau^{\lambda}\right)(\mu-1)\left(\frac{2 M}{\left(b_{1}-b_{2}-\frac{2 b_{4}(p-\lambda)}{p}\right) \mu}\right)^{\frac{\mu}{\mu-1}}+2 k C_{0} \\
= & \min \left\{\frac{1}{p}-b_{3} \tau^{p}-\frac{2 b_{4} \lambda}{p} \tau^{\lambda}, \frac{1}{2}\left(b_{1}-b_{2}-\frac{2 b_{4}(p-\lambda)}{p} \tau^{\lambda}\right)\right\} \frac{\rho-\frac{\mu-1}{\mu} T^{\frac{1-\mu}{\mu}}-\frac{T(p-1)}{p}}{\max \left\{\frac{1}{\mu}, \frac{1}{p}\right\}} \\
& -\frac{1}{2}\left(b_{1}-b_{2}-\frac{2 b_{4}(p-\lambda)}{p} \tau^{\lambda}\right)(\mu-1)\left(\frac{2 M}{\left(b_{1}-b_{2}-\frac{2 b_{4}(p-\lambda)}{p}\right) \mu}\right)^{\frac{\mu}{\mu-1}}+2 k C_{0},
\end{aligned}
$$

which together with (1.5) yields

$$
\varphi_{k}(u)>2 k C_{0}=\varphi_{k}(0) \quad \text { for all } u \in \partial \Omega \text {. }
$$

Thus by using Lemma 2.4 , we see that for each $k \in \mathbf{N}$, there is a point

$$
u_{k} \in \Omega_{1}:=\left\{x \in E_{k}: \int_{-k T}^{k T}\left|u^{\prime}(t)\right|^{p} d t+\int_{-k T}^{k T}|u(s)|^{\mu} d s<\rho_{1}\right\}
$$

such that

$$
\varphi_{k}\left(u_{k}\right)=\inf _{u \in \Omega} \varphi(u)
$$

In view of $\Omega_{1}$ being an open subset of $E_{k}$, we see from Theorem 1.3 in [12] that

$$
\varphi_{k}^{\prime}\left(u_{k}\right)=0
$$

and from (2.3) and the fact $u_{k} \in \Omega_{1}$, we see

$$
\int_{-k T}^{k T}\left|u_{k}^{\prime}(t)\right|^{p} d t+\int_{-k T}^{k T}\left|u_{k}(s)\right|^{\mu} d s<\frac{\rho-\frac{\mu-1}{\mu} T^{\frac{1-\mu}{\mu}}-\frac{T(p-1)}{p}}{\max \left\{\frac{1}{\mu}, \frac{1}{p}\right\}} .
$$

The proof is complete.

Lemma 2.6 Assume that assumption $\left(\mathrm{C}_{1}\right)$ of Theorem 1.2 , assumptions $\left(\mathrm{D}_{2}\right)-\left(\mathrm{D}_{3}\right)$ of Theorem 1.3 and condition (1.9) hold. Then for each $k \in \mathbf{N},(1.4)$ possesses a $2 k T$-periodic solution $u_{k} \in E_{k}$ such that

$$
\left\|u_{k}\right\|_{E_{k}}<\rho\left(T^{1-q}+T\right)^{-\frac{1}{q}}
$$

where $\rho>0$ is a constant determined by $\left(\mathrm{D}_{2}\right)$ and (1.9). Clearly, $\rho$ is independent of $k$.

Proof Let $\Gamma:=\left\{x \in E_{k}:\|x\|_{E_{k}} \leq \rho\left(T^{1-q}+T\right)^{-\frac{1}{q}}\right\}$. Clearly, $\Gamma$ is a bounded closed convex subset of $E_{k}$. Similar to the proof of Lemma 2.5, it suffices to show that for each $k \in \mathbf{N}$,

$$
\varphi_{k}(u)>\varphi_{k}(0), \quad \forall u \in \partial \Gamma
$$

If $u \in \partial \Gamma$, then $\|u\|_{E_{k}}=\rho\left(T^{1-q}+T\right)^{-\frac{1}{q}}$. So by using Lemma 2.3, we have

$$
\|u\|_{L_{2 K T}^{\infty}} \leq\left(T^{1-q}+T\right)^{\frac{1}{q}}\|u\|_{E_{k}}=\left(T^{1-q}+T\right)^{\frac{1}{q}} \rho\left(T^{1-q}+T\right)^{-\frac{1}{q}}=\rho .
$$


Furthermore, for all $u \in \partial \Gamma$, by using assumptions $\left(D_{2}\right)$ and $\left(D_{3}\right)$, and arguing in a similar way to the proof of Lemma 2.5 , we have

$$
\begin{aligned}
\varphi_{k}(u)= & \int_{-k T}^{k T}\left[\frac{1}{p}\left|u^{\prime}(t)\right|^{p}+G(t, u(t), u(t+\tau))+\left(e_{k}(t), u(t)\right)\right] d t \\
\geq & \int_{-k T}^{k T}\left[\frac{1}{p}\left|u^{\prime}(t)\right|^{p}+G(t, 0,0)+\left(b_{1}-b_{2}\right)|u(t)|^{p}-b_{3}|u(t)-u(t+\tau)|^{p}\right. \\
& \left.-b_{4}|u(t)-u(t+\tau)|^{\lambda}\left(|u(t)|^{(p-\lambda)}+|u(t+\tau)|^{(p-\lambda)}\right)+\left(e_{k}(t), u(t)\right)\right] d t \\
\geq & 2 k C_{0}+\int_{-k T}^{k T}\left[\frac{1}{p}\left|u^{\prime}(t)\right|^{p}+\left(b_{1}-b_{2}\right)|u(t)|^{p}+\left(e_{k}(t), u(t)\right)\right] d t \\
& -b_{3} \int_{-k T}^{k T}|u(t)-u(t+\tau)|^{p} d t \\
& -2 b_{4}\left(\int_{-k T}^{k T}|u(t)-u(t+\tau)|^{p} d t\right)^{\frac{\lambda}{p}}\left(\int_{-k T}^{k T}|u(t)|^{p} d t\right) t^{\frac{p-\lambda}{p}}-M_{1}\|u\|_{p} \\
\geq & \min \left\{\frac{1}{p}-b_{3} \tau^{p}-\frac{2 b_{4} \lambda}{p} \tau^{\lambda}, \frac{1}{2}\left(b_{1}-b_{2}-\frac{2 b_{4}(p-\lambda)}{p} \tau^{\lambda}\right)\right\}\left(\|u\|_{p}^{p}+\left\|u^{\prime}\right\|_{p}^{p}\right) \\
& -\frac{1}{2}\left(b_{1}-b_{2}-\frac{2 b_{4}(p-\lambda)}{p} \tau^{\lambda}\right)(p-1)\left(\frac{2 M_{1}}{\left(b_{1}-b_{2}-\frac{2 b_{4}(p-\lambda)}{p}\right) p}\right)^{\frac{p}{p-1}}+2 k C_{0} \\
= & \min \left\{\frac{1}{p}-b_{3} \tau^{p}-\frac{2 b_{4} \lambda}{p} \tau^{\lambda}, \frac{1}{2}\left(b_{1}-b_{2}-\frac{2 b_{4}(p-\lambda)}{p} \tau^{\lambda}\right)\right\} \rho\left(T^{1-q}+T\right)^{-\frac{1}{q}} \\
& -\frac{1}{2}\left(b_{1}-b_{2}-\frac{2 b_{4}(p-\lambda)}{p} \tau^{\lambda}\right)(p-1)\left(\frac{2 M_{1}}{\left(b_{1}-b_{2}-\frac{2 b_{4}(p-\lambda)}{p}\right) p}\right)^{\frac{p}{p-1}}+2 k C_{0},
\end{aligned}
$$

which together with (1.9) yields

$$
\varphi_{k}(u)>2 k C_{0}=\varphi_{k}(0) \quad \text { for all } u \in \partial \Gamma \text {. }
$$

The proof is complete.

Lemma 2.7 [7] Let $u_{k} \in E_{k}$ be the $2 k T$-periodic solution to (1.4) that satisfies (2.1) for each $k \in \mathbf{N}$. Then there exists a subsequence $\left\{u_{k_{j}}\right\}$ of $\left\{u_{k}\right\}$ convergent to a $u_{0} \in C^{1}\left(R, R^{n}\right)$ in $C_{\mathrm{loc}}^{1}\left(R, R^{n}\right)$.

\section{Proof of main result}

Proof of Theorem 1.2 Firstly, we will prove that $u_{0}(t)$, which is determined by Lemma 2.7, is a solution to (1.3). Since $u_{k_{j}}(t)$ is a $2 k T$-periodic solution to (1.4), it follows that

$$
\begin{aligned}
& \frac{d}{d t}\left(\left|u_{k_{j}}^{\prime}(t)\right|^{p-2} u_{k_{j}}^{\prime}(t)\right) \\
& \quad=\nabla_{x} G\left(t, u_{k_{j}}(t), u_{k_{j}}(t+\tau)\right)+\nabla_{y} G\left(t-\tau, u_{k_{j}}(t-\tau), u_{k_{j}}(t)\right)+e_{k_{j}}(t), \quad j \in \mathbf{N} .
\end{aligned}
$$

Take $a, b \in R$ with $a<b$, then there must be a positive integer $j_{0}$ such that for $j>j_{0}$, $\left[-k_{j} T, k_{j} T\right] \supset[a-\tau, b+\tau]$. So for $j>j_{0}, e_{k_{j}}(t)=e(t)$ for all $t \in[a-\tau, b+\tau]$, and then 
by (3.1)

$$
\begin{aligned}
& \frac{d}{d t}\left(\left|u_{k_{j}}^{\prime}(t)\right|^{p-2} u_{k_{j}}^{\prime}(t)\right) \\
& \quad=\nabla_{x} G\left(t, u_{k_{j}}(t), u_{k_{j}}(t+\tau)\right)+\nabla_{y} G\left(t-\tau, u_{k_{j}}(t-\tau), u_{k_{j}}(t)\right)+e(t), \quad t \in[a, b], j>j_{0} .
\end{aligned}
$$

Thus, by using Lemma 2.7, $\frac{d}{d t}\left[\left|u_{k_{j}}^{\prime}(t)\right|^{p-2} u_{k_{j}}^{\prime}(t)\right] \rightarrow w(t)$ uniformly for $t \in[a, b]$, where

$$
w(t)=\nabla_{x} G\left(t, u_{0}(t), u_{0}(t+\tau)\right)+\nabla_{y} G\left(t-\tau, u_{0}(t-\tau), u_{0}(t)\right)+e(t) .
$$

Since $\left|u_{k_{j}}^{\prime}(t)\right|^{p-2} u_{k_{j}}^{\prime}(t) \rightarrow\left|u_{0}^{\prime}(t)\right|^{p-2} u_{0}^{\prime}(t)$ for $t \in[a, b]$ and $\frac{d}{d t}\left(\left|u_{k_{j}}^{\prime}(t)\right|^{p-2} u_{k_{j}}^{\prime}(t)\right)$ is continuous differential of $\left|u_{k_{j}}^{\prime}(t)\right|^{p-2} u_{k_{j}}^{\prime}(t)$ on $(a, b)$ for every $j>j_{0}$, it follows that $w(t)=$ $\frac{d}{d t}\left[\left|u_{0}^{\prime}(t)\right|^{p-2} u_{0}^{\prime}(t)\right]$ on $(a, b)$. In view of $a, b \in R$ being arbitrary with $a<b, w(t)=$ $\frac{d}{d t}\left[\left|u_{0}^{\prime}(t)\right|^{p-2} u_{0}^{\prime}(t)\right], t \in R$, that is, $u_{0}(t), t \in R$ is a solution to (1.5).

Below, we will prove $u_{0}(t) \rightarrow 0$ and $u_{0}^{\prime}(t) \rightarrow 0$ as $|t| \rightarrow+\infty$.

Since

$$
\begin{aligned}
\int_{-\infty}^{+\infty}\left(\left|u_{0}(t)\right|^{\mu}+\left|u_{0}^{\prime}(t)\right|^{p}\right) d t & =\lim _{i \rightarrow+\infty} \int_{-i T}^{i T}\left(\left|u_{0}(t)\right|^{\mu}+\left|u_{0}^{\prime}(t)\right|^{p}\right) d t \\
& =\lim _{i \rightarrow+\infty} \lim _{j \rightarrow+\infty} \int_{-i T}^{i T}\left(\left|u_{k_{j}}(t)\right|^{\mu}+\left|u_{k_{j}}^{\prime}(t)\right|^{p}\right) d t
\end{aligned}
$$

clearly, for every $i \in \mathbf{N}$ if $k_{j}>i$, by (2.1),

$$
\begin{aligned}
\int_{-i T}^{i T}\left(\left|u_{k_{j}}(t)\right|^{\mu}+\left|u_{k_{j}}^{\prime}(t)\right|^{p}\right) d t & \leq \int_{-k_{j} T}^{k_{j} T}\left(\left|u_{k_{j}}(t)\right|^{\mu}+\left|u_{k_{j}}^{\prime}(t)\right|^{p}\right) d t \\
& \leq A_{0}^{\mu}+A_{1}^{p} .
\end{aligned}
$$

Let $i \rightarrow+\infty$ and $j \rightarrow+\infty$, respectively, we have

$$
\int_{-\infty}^{+\infty}\left(\left|u_{0}(t)\right|^{\mu}+\left|u_{0}^{\prime}(t)\right|^{p}\right) d t \leq A_{0}^{\mu}+A_{1}^{p}
$$

and then

$$
\int_{|t| \geq r}\left|u_{0}(t)\right|^{\mu} d t \rightarrow 0, \quad \int_{|t| \geq r}\left|u_{0}^{\prime}(t)\right|^{p} d t \rightarrow 0 \quad \text { as } r \rightarrow+\infty .
$$

So by using Lemma 2.3 ,

$$
\begin{aligned}
\left|u_{0}(t)\right| & \leq(2 T)^{-1 / \mu}\left(\int_{t-T}^{t+T}\left|u_{0}(s)\right|^{\mu} d s\right)^{1 / \mu}+T(2 T)^{-1 / p}\left(\int_{t-T}^{t+T}\left|u_{0}^{\prime}(s)\right|^{p} d s\right)^{1 / p} \\
& \rightarrow 0, \quad \text { as }|t| \rightarrow+\infty
\end{aligned}
$$

Thus, $\left\|u_{0}\right\|_{\infty} \leq \rho_{0}<+\infty$, which, together with the fact that $u_{0}(t)$ is a solution of (1.3), i.e.,

$$
\frac{d}{d t}\left[\left|u_{0}^{\prime}(t)\right|^{p-2} u_{0}^{\prime}(t)\right]=\nabla_{x} G\left(t, u_{0}(t), u_{0}(t+\tau)\right)+\nabla_{y} G\left(t-\tau, u_{0}(t-\tau), u_{0}(t)\right)+e(t),
$$


yields

$$
\begin{aligned}
\left|\frac{d}{d t}\left[\left|u_{0}^{\prime}(t)\right|^{p-2} u_{0}^{\prime}(t)\right]\right| & \leq \max _{t \in[0, T],|x|,|y| \leq \rho_{0}}\left|\nabla_{x} G(t, x, y)+\nabla_{y} G(t-\tau, x, y)\right|+\sup _{t \in R}|e(t)| \\
& :=R_{1}<+\infty .
\end{aligned}
$$

Furthermore, from (3.2), $\int_{-\infty}^{+\infty}\left|u_{0}^{\prime}(t)\right|^{p} d t \leq A_{0}^{\mu}+A_{1}^{p}$; and then by using Lemma 2.1, we have

$$
\left|u_{0}^{\prime}(t)\right| \rightarrow 0, \quad \text { as }|t| \rightarrow+\infty
$$

Combining (3.3) and (3.4), we see $u_{0}(t)$ is a homoclinic solution to (1.3). Clearly, $u_{0}(t) \not \equiv 0$, otherwise, by substituting $u_{0}(t) \equiv 0$ into (1.3), we have

$$
\nabla_{x} G(t, 0,0)+\nabla_{y} G(t-\tau, 0,0) \equiv e(t) .
$$

By using assumption $\left(\mathrm{C}_{1}\right)$, we get $\nabla_{x} G(t, 0,0)+\nabla_{y} G(t-\tau, 0,0)=0$ for all $t \in R$. So it follows from (3.5) that $e(t) \equiv 0$, which contradicts the fact that $e(t) \not \equiv 0$ in assumption $\left(C_{3}\right)$. The proof is complete.

Since the proof of Theorem 1.3 works almost exactly as the proof of Theorem 1.2, we omit the proof of Theorem 1.3 here.

For example, consider the following equation:

$$
\begin{aligned}
u^{\prime \prime}(t)= & 31.5 u^{3}(t)-5 u^{4}(t)-2 u^{2}(t+\tau)+4 u(t) u(t+\tau)+2 u^{2}(t-\tau) \\
& -4 u(t) u(t-\tau)-4 u(t)+2 u(t+\tau)+2 u(t-\tau)+e(t),
\end{aligned}
$$

where $x(t) \in R, e(t)=\frac{e^{\frac{t}{2}}}{2\left(e^{t}+e^{-t}\right)}$, and $\tau>0$ is a constant.

By calculating, we can choose $G(t, x, y)=8 x^{4}-\frac{1}{8} y^{4}-x^{5}-(x-y)^{2}-2(x-y)\left(x^{2}+y^{2}\right)+\sin t$ such that (3.6) is rewritten as

$$
u^{\prime \prime}(t)=\frac{\partial G(t, u(t), u(t+\tau))}{\partial x}+\frac{\partial G(t-\tau, u(t-\tau), u(t))}{\partial y}+e(t) .
$$

Corresponding to Theorem 1.2, we see $p=2, T=2 \pi$. So we can choose $\mu=4, \lambda=1$, $c_{1}=1, b_{1}=8, b_{2}=2, b_{3}=1$ and $b_{4}=2$ such that conditions $\left(C_{1}\right)$ and $\left(C_{2}\right)$ are satisfied; and also

$$
M=\left(\int_{-\infty}^{+\infty}\left(\frac{e^{\frac{t}{2}}}{2\left(e^{t}+e^{-t}\right)}\right)^{3 / 4} d t\right)^{4 / 3}=2\left(\frac{3}{2}\right)^{3 / 4}<+\infty,
$$

which implies that condition $\left(\mathrm{C}_{3}\right)$ holds. So we can choose $\rho=7.75$ such that $\delta=b_{1}-\rho=$ $0.25>0$, and if $\tau>0$ is sufficiently small, then

$$
\delta-b_{2}-\frac{2 b_{4}(p-\lambda)}{p} \tau^{\lambda}>0
$$


and

$$
\begin{gathered}
\min \left\{\frac{1}{p}-b_{3} \tau^{p}-\frac{2 b_{4} \lambda}{p} \tau^{\lambda}, \frac{1}{2}\left(\delta-b_{2}-\frac{2 b_{4}(p-\lambda)}{p} \tau^{\lambda}\right)\right\} \frac{\rho-\frac{\mu-1}{\mu} T^{\frac{1-\mu}{\mu}}-\frac{T(p-1)}{p}}{\max \left\{\frac{1}{\mu}, \frac{1}{p}\right\}} \\
-\frac{1}{2}\left(\delta-b_{2}-\frac{2 b_{4}(p-\lambda)}{p} \tau^{\lambda}\right)(\mu-1)\left(\frac{2 M}{\left(\delta-b_{2}-\frac{2 b_{4}(p-\lambda)}{p} \tau^{\lambda}\right) \mu}\right)^{\frac{\mu}{\mu-1}}>0 .
\end{gathered}
$$

Thus, by using Corollary 1.1, we see that (3.6) has a nontrivial homoclinic solution for $\tau>0$ small enough.

Especially, if $\tau=0$, then (3.6) is converted to

$$
u^{\prime \prime}(t)=31.5 u^{3}(t)-5 u^{4}(t)+e(t)
$$

So we can choose $G(t, x, x)=7.825 x^{4}-x^{5}+\sin t$ such that (3.7) is written as

$$
u^{\prime \prime}(t)=\frac{\partial G(t, u(t), u(t))}{\partial x}+\frac{\partial G(t, u(t), u(t))}{\partial y}+e(t) .
$$

Clearly, we can choose $\rho=7.75$ such that all the conditions of Corollary 1.1 are satisfied. So (3.7) has a nontrivial homoclinic solution.

Remark 3.1 From (3.7), we see that if set $G(t, x, x)=F(t, x)$, then (1.1) is the special case of (3.6) for $\tau=0$. Also, from (3.7), we see that

$$
F(t, x)=G(t, x, x) \rightarrow-\infty, \quad \text { as }|x| \rightarrow+\infty,
$$

which implies that the crucial assumption $\left(B_{2}\right)$ for guaranteeing the coercive condition in [7] (see Theorem 1.1 in Section 1) does not hold. So the results in present paper are essentially new.

\section{Competing interests}

The authors declare that they have no competing interests.

\section{Authors' contributions}

All authors have equally contributed to obtaining new results in this article and also read and approved the final manuscript.

\section{Author details}

${ }^{1}$ College of Math and Statistics, Nanjing University of Information Science \& Technology, Nanjing, 210044, China.

${ }^{2}$ Department of Mathematics, Anhui Normal University, Wuhu, 241000, China.

\section{Acknowledgements}

Research supported by the NSF of China (No. 11271197).

Received: 8 April 2014 Accepted: 2 September 2014 Published: 24 Sep 2014

\section{References}

1. Lin, $X Y$, Tang, $X H$ : Infinitely many homoclinic orbits of second-order $p$-Laplacian systems. Taiwan. J. Math. 17(4), 1371-1393 (2013)

2. Zhang, Z, Yuan, R: Homoclinic solutions for a class of non-autonomous subquadratic second-order Hamiltonian systems. Nonlinear Anal. 71, 4125-4130 (2009)

3. Tan, $\mathrm{XH}$, Xiao, L: Homoclinic solutions for nonautonomous second-order Hamiltonian systems with a coercive potential. J. Math. Anal. Appl. 351, 586-594 (2009) 
4. Izydorek, M, Janczewska, J: Homoclinic solutions for nonautonomous second order Hamiltonian systems with a coercive potential. J. Math. Anal. Appl. 335, 1119-1127 (2007)

5. Rabinowitz, PH: Homoclinic orbits for a class of Hamiltonian systems. Proc. R. Soc. Edinb., Sect. A 114, 33-38 (1990)

6. Izydorek, M, Janczewska, J: Homoclinic solutions for a class of the second order Hamiltonian systems. J. Differ. Equ. 219, 375-389 (2005)

7. Tang, XH, Xiao, L: Homoclinic solutions for ordinary p-Laplacian systems with a coercive potential. Nonlinear Anal. 71, 1124-1132 (2009)

8. Lin, XB: Exponential dichotomies and homoclinic orbits in functional differential equations. J. Differ. Equ. 63, 227-254 (1986)

9. Guo, CJ, O'Regan, D, Xu, YT, Agarwal, RP: Homoclinic orbits for a singular second-order neutral differential equation. J. Math. Anal. Appl. 366, 550-560 (2010)

10. Lu, SP: Homoclinic solutions for a nonlinear second order differential system with $p$-Laplacian operator. Nonlinear Anal., Real World Appl. 12, 525-534 (2011)

11. Lu, SP: Sufficient conditions for the existence of periodic solutions to some second order differential equations with a deviating argument. J. Math. Anal. Appl. 308, 393-419 (2005)

12. Mawhin, JL, Willem, M: Critical Point Theory and Hamiltonian Systems. Springer, New York (1989)

10.1186/1687-1847-2014-244

Cite this article as: Lu and Lu: Homoclinic solutions for a second-order $p$-Laplacian functional differential system with local condition. Advances in Difference Equations 2014, 2014:244

\section{Submit your manuscript to a SpringerOpen ${ }^{\ominus}$ journal and benefit from:}

- Convenient online submission

- Rigorous peer review

- Immediate publication on acceptance

- Open access: articles freely available online

- High visibility within the field

- Retaining the copyright to your article 